\title{
COMPARATIVE EFFECTIVENESS STUDY OF COMBINED ANTIHYPERTENSIVES FOR NIGERIAN PATIENTS
}

\section{CHINWE VICTORIA UKWE ${ }^{1}$, MAXWELL OGOCHUKWU ADIBE ${ }^{1 *}$, OKAFOR CE ${ }^{2}$, ANOSIKE $\mathrm{C}^{1}$, ISAH A ${ }^{1}$, IKE SO ${ }^{3}$, ANISIUBA BC ${ }^{3}$, NWURUKU GC ${ }^{4}$}

${ }^{1}$ Department of Clinical Pharmacy and Pharmacy Management, Faculty of Pharmaceutical Sciences, University of Nigeria, Nsukka. ${ }^{2}$ Department of Clinical Pharmacy and Pharmacy Management, Faculty of Pharmaceutical Sciences, Nnamdi Azikiwe University, Awka. ${ }^{3}$ Cardiology Unit, Department of Medicine, College of Medicine, University of Nigeria, Enugu Campus. ${ }^{4}$ Department of Medicine, Alex Ekwueme Federal University Teaching Hospital, Abakaliki, Ebonyi State. Email: maxwell.adibe@unn.edu.ng

Received: 10 March 2020, Revised and Accepted: 15 April 2020

ABSTRACT

Objective: The objective of the study was to compare the clinical and economic effectiveness of four combination antihypertensives recommended for Nigerians.

Methods: An open, randomized, controlled, and longitudinal double-blind trial of four groups of antihypertensives combinations: Telmisartan/ chlorthalidone/amlodipine (TCA), TC, CA, and TA was conducted among hypertensive patients. The participants were recruited from three hospitals in Enugu, and randomly assigned to the study groups. The primary outcome for this study was blood pressure (BP) control, based on Joint National Committee-8 and cost per BP control. The secondary outcomes were cost per quality adjusted life years (QALY) and patients' self-reported health status. Descriptive and inferential statistics were used for statistical analysis.

Results: Of the 110 patients enrolled in the study, more than half were women (55.5\%). The mean age of patients was $54.93 \pm 12.38$. The enrollees had hypertension for over 9 years $(9.17 \pm 8.40)$. About $77 \%$ of the patients completed the study in all the groups except for TA $(66.7 \%)$. There was no difference in BP in all the groups at baseline and at end-of-study ( $\mathrm{p}>0.050)$. However, the probability of BP control was highest in TCA group $(0.37 \pm 0.01)$, followed by TC group $(0.23 \pm 0.02)$. The TA group showed the most favorable cost per QALY, then CA, TC, and TCA in that order. The group with the most favorable cost per BP control was TCA (70.92 \pm 0.04$)$, then TA (94.16 \pm 0.05$)$.

Conclusion: The triple combination therapy of TCA had the best cost per BP control in the management of hypertensive patients. It demonstrated the highest probability of BP control.

Keywords: Antihypertensive medications, Combination therapy, Cost-effectiveness, Health status.

(C) 2020 The Authors. Published by Innovare Academic Sciences Pvt Ltd. This is an open access article under the CC BY license (http://creativecommons. org/licenses/by/4. 0/) DOI: http://dx.doi.org/10.22159/ajpcr.2020.v13i6.37399

\section{INTRODUCTION}

Hypertension is still a global public health problem, and the financial cost associated with the disease continues to increase. It is estimated to affect over 1 billion people worldwide [1]. Sub-Saharan Africa (SSA) including Nigeria, account for approximately two-thirds of the global burden of hypertension. Hypertension is a major risk factor for the development of other cardiovascular diseases such as heart failure, stroke, and ischemic heart disease [2]. It is one of the leading causes of morbidity and mortality due to cardiovascular complications $[3,4]$.

Nigeria presently has a population estimate of over 180 million, and thus the most populous country in Africa. In 2014, hypertension prevalence in Nigeria was estimated at $28.9 \%$ by the World Health Organization. However, in 2030, it is projected that the country will record 39.1 million cases of hypertension among its adult population, with a prevalence of $30.8 \%$ [5,6]. Therefore, Nigeria contributes significantly to the hypertension burden in SSA. The high prevalence of hypertension in Nigeria is probably the result of increasing aging population, rapid urbanization, high intake of processed food, tobacco, and alcoholic products [7]. In addition, treatment and control of hypertension are low in Nigeria and other countries in SSA. For instance, a study reported that only $25.4 \%$ of patients attending a hypertension clinic in a tertiary hospital achieved desired blood pressure (BP) control [8].

Research has demonstrated that it is usually difficult achieving BP control with a single antihypertensive medication $[9,10]$. Evidently, about $75 \%$ of patients with hypertension would require two or more antihypertensive drug combination to ensure optimal BP control. This is because the specific cause of hypertension is rarely known and BP elevation is usually the product of multiple factors [9-11]. This makes it almost impossible to control BP with administration of a single antihypertensive medication.

Nevertheless, the Joint National Committee (JNC) on the prevention, detection, evaluation, and treatment of high BP (JNC-8), recommends monotherapy for initial treatment of hypertension in persons $<60$ years with BP above $140 / 90 \mathrm{mmHg}$, with or without diabetes, or chronic kidney disease (CKD). The BP target for patients aged 60 years and above was fixed at $150 / 90 \mathrm{mmHg}$. According to the guideline, failure to attain BP target with a single agent warrants the addition of one or more antihypertensive drugs with different mechanisms of action, after titrating initial therapy to a maximum dose, if necessary [12].

In general, hypertension is incurable. Hence, its treatment is lifelong. There are several available antihypertensive drugs for optimal BP control, with varying clinical effectiveness, cost implication, and side effect profile $[13,14]$. Some of the antihypertensive medications have proven efficacy, but are not affordable by majority of the populace.

However, there appears to be limited data comparing the efficacy and cost of antihypertensive combination therapies to determine the most suitable for black patients in Nigeria and sub-Sahara Africa in general. Therefore, this study aimed to compare the BP control and 
cost-effectiveness of four different combinations of antihypertensive therapies recommended for black Africans $[12,15,16]$.

\section{METHODS}

\section{Study design}

An open, randomized, controlled, longitudinal double-blind, and fourarm parallel prospective trial with 6 weeks patient follow-up. The four arms of the study were: (1) Amlodipine + Telmisartan (AT), (2) A + Chlorthalidone (TAC), (3) $\mathrm{T}+\mathrm{C}$, and (4) A + T + C.

\section{Study setting}

This study was conducted in three private hospitals in Enugu. The facilities used were the Redemption Clinic, Kenyatta Hospital, and Doctor-on-call Clinic.

\section{Study population}

The study participants consist of 110 hypertensive patients who are 18 years and above. The participants were recruited from the outpatient clinics of the study centers.

\section{Patients' allocation}

The patients were allocated to the four arms of the study through a mixture of cluster and random sampling techniques. The patients in each clinic who met the inclusion criteria were distributed into clusters based on their matching characteristics. Subsequently, they were allocated to any of the four study arms through simple random technique. The criteria for grouping patients into clusters were age (18-59 years, $\geq 60$ years), sex (male, female), and severity/comorbidity (CKD and/or diabetes, no CKD nor diabetes). Thus, giving rise to eight independent clusters.

\section{Eligibility criteria}

The study participants were recruited based on the following inclusion criteria: (1) Patients who are 18 years and above, (2) patients with compelling indications to use a combination therapy, and (3) patients who accepted to visit the clinic weekly for BP measurement and medication refill. The study excluded pregnant women.

\section{Study instruments}

The patients' socio-demographic and clinical characteristics were obtained using a pro forma designed for such purpose. The sociodemographic data retrieved include patients' study group, age, sex, marital status, place of residence, occupation, monthly income, and educational level. The clinical variables were duration of hypertension since diagnosis, smoking and alcohol status, physical activity, use of herbal/traditional medicines, and adjuvant medications. Others are BP weekly readings, urine tests (e.g., urine protein, glucose, ketone, $\mathrm{PH}$, nitrite, creatinine clearance, $\mathrm{HCO}_{3}$, and $\mathrm{Tc}_{\mathrm{a}}{ }^{2+}$ ), blood tests (e.g., packed cell volume, bilirubin, glucose, fasting blood sugar, creatinine, lowdensity lipoprotein, high-density lipoprotein, and triglycerides), and anthropometrics (e.g., height, weight, and hip circumference).

\section{Data collection}

The patients were assigned to four study groups. Each patient received a 6 weeks supply of antihypertensive drug combinations based on their group. Neither the physician nor the patients knew whether they received a triple combination antihypertensive or any of the three dual combinations. The patients were encouraged to take the medications daily and to monitor their BP. They were asked not to use any other antihypertensive medications throughout the study period of 6 weeks, and to report to the physician if there was a need to stop therapy. The patients were required to visit the clinic once a week, where the physicians examined their health status, measured, and recorded their $\mathrm{BP}$ and other clinical information.

The EQ-5D-5L English language version was used to measure patients' health status. The instrument was developed and validated by the EuroQol group. It is internationally accepted and recommended for use in economic evaluation studies. The previous studies have re-validated the instrument among Nigerian population [17]. The EQ-5D instrument consists of the descriptive system and the EQ visual analogue scale (EQVAS). The descriptive system had five dimensions: Mobility, usual care, self-care, anxiety/depression, and pain/discomfort. Each dimension had five levels: "(1) No problem, (2) slight problem, (3) moderate problem, (4) severe problem, and (5) extreme problem." On the other hand, the EQ-VAS is a $20 \mathrm{~cm}$ scale with endpoints ranging from 0 to 100 . The endpoints " 0 " and " 100 " signify "the worst health you can imagine" and "the best health you can imagine," respectively. A box for recording patients' self-rated health state was provided beside the scale.

\section{Outcome measures}

The primary outcome for this study was BP control based on JNC-8 guideline and cost per BP control, while the secondary outcomes were cost per quality adjusted life years (QALY) and patients' self-reported health status.

\section{Statistical analysis}

The patients' socio-demographic, clinical characteristics, and health status were presented using descriptive statistics (frequency, percent, mean, and standard deviation). Student t-test was employed in examining the mean difference between the pre- and post-tests continuous laboratory values assessed across various study groups. One-way ANOVA test was used to determine the mean difference in systolic BP and diastolic BP for the different study groups. QALY was calculated using the average utility valuation score for the study population. ${ }^{*} \mathrm{p}<0.05$ was considered to be statistically significant.

\section{Ethical considerations}

Ethical Committee of University of Nigeria Teaching approved the protocol of study. The patients who are willing to participate were provided with additional written information and asked to sign the study consent form or thumb print for illiterate patients.

\section{RESULTS}

Table 1 shows the socio-demographic and clinical characteristics of the patients. Out of the 110 patients enrolled in the study, more than half were women (55.5\%). The mean age of patients was $54.93 \pm 12.38$. The majority of the patients were married (78.2\%) and are urban dwellers $(82.7 \%)$. Less than half of the participants had tertiary education (44.5\%). On average, the enrollees have had hypertension for over 9 years $(9.17 \pm 8.40)$.

Table 2 reveals the distribution of patients with controlled BP across the different study groups. At the end of study, patients on CA antihypertensive combination had the highest proportion of patients with BP control (83.3\%), while enrollees on TA had the least BP control $(54.2 \%)$

Table 3 shows the probability of BP control and cost-effectiveness of a triple antihypertensive combination and three dual combinations. The results showed that patients' reported health status indices (EQVAS and EQ-5D index scores) were highest for CA group, followed by TCA group. The probability of BP control was highest in TCA group $(0.37 \pm 0.001)$, while the least was the TA group $(0.19 \pm 0.001)$. The TA group demonstrated the best cost per QALY, followed by CA, TC, and TCA in that order. However, the group with the most favorable cost per BP control was TCA $(70.92 \pm 0.04)$, followed by TA $(94.16 \pm 0.05)$.

Figs. 1 and 2 shows the acceptability curves for the different interventions. TCA and TA had the best probabilities of being costeffective at competitive willingness to pay values.

\section{DISCUSSION}

This study was the first that compared the effectiveness of combined antihypertensive medications in terms of the BP control and QALY among patients in Nigeria and SSA. Its major findings demonstrate that the probability of $\mathrm{BP}$ control was highest among patients on the triple 
Table 1: Socio-demographic and clinical characteristics of the patients

\begin{tabular}{|c|c|c|c|c|c|}
\hline \multirow[t]{2}{*}{ Variable } & \multicolumn{5}{|c|}{ Frequency (percent) } \\
\hline & TCA & TC & CA & TA & Total \\
\hline Age (years) & $54.12 \pm 12.15$ & $57.80 \pm 10.78$ & $53.67 \pm 14.75$ & $54.29 \pm 12.77$ & $54.93 \pm 25.39 *$ \\
\hline \multicolumn{6}{|l|}{ Sex } \\
\hline Male & $19(45.2)$ & $13(50.0)$ & $6(33.3)$ & $11(45.8)$ & $49(44.5)$ \\
\hline Female & $23(54.8)$ & $13(50.0)$ & $12(66.7)$ & $13(54.2)$ & $61(55.5)$ \\
\hline \multicolumn{6}{|l|}{ Marital status } \\
\hline Single & $4(9.5)$ & $2(7.7)$ & $1(5.6)$ & $0(0.0)$ & $7(6.4)$ \\
\hline Married & $32(76.2)$ & $20(76.9)$ & $15(83.3)$ & $19(79.2)$ & $86(78.2)$ \\
\hline Widowed & $5(11.9)$ & $4(15.4)$ & 2 (11.1) & $5(20.8)$ & $16(14.5)$ \\
\hline \multicolumn{6}{|l|}{ Place of residence } \\
\hline Urban & 33 (78.6) & $22(84.6)$ & $16(88.9)$ & $20(83.3)$ & $91(82.7)$ \\
\hline Rural & 5 (11.9) & $2(7.7)$ & $2(11.1)$ & $2(8.3)$ & $11(10.0)$ \\
\hline \multicolumn{6}{|l|}{ Occupation } \\
\hline Unemployed & $6(14.3)$ & $6(23.1)$ & $6(33.3)$ & $4(16.7)$ & $22(20.0)$ \\
\hline Employed & $22(52.4)$ & $13(50.0)$ & $7(38.9)$ & $9(37.5)$ & $51(46.4)$ \\
\hline Retired & $11(26.2)$ & $7(26.9)$ & $5(27.8)$ & $7(29.2)$ & $30(27.3)$ \\
\hline Student & $1(2.4)$ & $0(0.0)$ & $0(0.0)$ & $0(0.0)$ & $1(0.9)$ \\
\hline \multicolumn{6}{|l|}{ Educational level } \\
\hline None & $3(7.1)$ & $1(3.8)$ & $1(5.6)$ & $2(8.3)$ & $7(6.4)$ \\
\hline Primary & $10(23.8)$ & 7 (26.9) & $3(16.7)$ & $8(33.3)$ & $28(25.5)$ \\
\hline Secondary & $12(28.6)$ & $3(11.5)$ & 3 (16.7) & $5(20.8)$ & $23(20.9)$ \\
\hline Tertiary & $16(38.1)$ & $14(53.8)$ & $11(61.1)$ & $8(33.3)$ & $49(44.5)$ \\
\hline Monthly income (naira) & $47933 \pm 69733$ & $75318 \pm 12697$ & $27125 \pm 24879$ & $35053 \pm 34858$ & $49190 \pm 34858^{*}$ \\
\hline Duration of diagnosis & $10.27 \pm 9.303$ & $9.56 \pm 8.917$ & $9.35 \pm 8.403$ & $6.96 \pm 6.175$ & $9.17 \pm 16.577^{*}$ \\
\hline \multicolumn{6}{|l|}{ Comorbidity } \\
\hline Diabetes & $0(0.0)$ & $4(15.4)$ & $2(11.1)$ & $2(8.3)$ & $8(7.3)$ \\
\hline Hyperlipidemia & $4(9.5)$ & $1(3.8)$ & $1(5.6)$ & $1(4.2)$ & $2(1.8)$ \\
\hline Stroke & $1(2.4)$ & $1(3.8)$ & $2(11.1)$ & $1(4.2)$ & $8(7.3)$ \\
\hline Others & $5(11.9)$ & $1(3.8)$ & $1(5.6)$ & $2(8.3)$ & $9(8.2)$ \\
\hline \multicolumn{6}{|l|}{ Smoking status } \\
\hline No & $40(95.2)$ & $25(96.2)$ & $16(88.9)$ & $22(91.7)$ & $103(93.6)$ \\
\hline Yes & $2(4.8)$ & $0(0.0)$ & $0(0.0)$ & $1(4.2)$ & $3(2.7)$ \\
\hline \multicolumn{6}{|l|}{ Alcohol status } \\
\hline No & $28(66.7)$ & $14(53.8)$ & $11(61.1)$ & $14(58.3)$ & $67(60.9)$ \\
\hline Yes & $14(33.3)$ & $12(46.2)$ & $7(38.9)$ & $9(37.5)$ & $42(38.2)$ \\
\hline \multicolumn{6}{|c|}{ Herbal/traditional medicine status } \\
\hline No & 33 (78.6) & $21(80.8)$ & $12(66.7)$ & $19(79.2)$ & $85(77.3)$ \\
\hline Yes & $9(21.4)$ & $5(19.2)$ & $6(33.3)$ & $4(16.7)$ & $24(21.8)$ \\
\hline \multicolumn{6}{|l|}{ BP apparatus } \\
\hline No & $28(66.7)$ & $16(61.5)$ & $12(66.7)$ & $16(66.7)$ & $72(65.5)$ \\
\hline Yes & $14(33.3)$ & $10(38.5)$ & $6(33.3)$ & $7(29.2)$ & 37 (33.6) \\
\hline \multicolumn{6}{|l|}{ Physical activity } \\
\hline No & $10(23.8)$ & $5(19.2)$ & $5(27.8)$ & $9(37.5)$ & $29(26.4)$ \\
\hline Yes & 31 (73.8) & $20(76.9)$ & $13(72.2)$ & $13(54.2)$ & $77(70.0)$ \\
\hline
\end{tabular}

*Mean \pm standard deviation. TCA: Telmisartan/chlorthalidone/amlodipine, TC: Telmisartan/chlorthalidone, CA: Chlorthalidone/amlodipine, TA: Telmisartan/amlodipine. TCA: Telmisartan/chlorthalidone/amlodipine, TC: Telmisartan/chlorthalidone, CA: Chlorthalidone/amlodipine, TA: Telmisartan/amlodipine, BP: Blood pressure

Table 2: Blood pressures control across the different study groups

\begin{tabular}{lll}
\hline \multirow{2}{*}{ Study groups } & \multicolumn{2}{l}{ BP control, n (\%) } \\
\cline { 2 - 3 } & Uncontrolled & Controlled \\
\hline $\begin{array}{l}\text { Telmisartan+Chlorthalidone } \\
\text { +Amlodipine }\end{array}$ & $16(38.1)$ & $26(61.9)$ \\
Telmisartan+Chlorthalidone & $10(38.5)$ & $16(61.5)$ \\
Chlorthalidone+Amlodipine & $3(16.7)$ & $15(83.3)$ \\
Telmisartan+Amlodipine & $11(45.8)$ & $13(54.2)$ \\
\hline
\end{tabular}

combination (TCA) therapy, followed sequentially by patients on TC, CA, and TA. It also showed that TCA had the most favorable cost per BP control followed by TA. Other findings showed that patients on TA combination had the best cost per QALY.

Acceptability curves were used to present the result of costeffectiveness analysis. Acceptability curves illustrate the probability that any particular intervention is cost-effective conditional on the willingness to pay per QALY [18]. The curve also illustrates the degree of uncertainty in the estimates. The cost-effectiveness acceptability curve based on BP control showed that at willingness to pay above $\$ 40$, TCA was the most cost-effective. At higher willingness to pay values, no other combination was a contender for cost-effectiveness with TCA. At willingness to pay below $\$ 40$, TA was the most cost-effective.

The cost-effectiveness acceptability curve based of QALY showed that at willingness to pay below $\$ 600$, TA was the most cost-effective. At willingness to pay above $\$ 600$, CA and TCA were the most effective. Even at willingness to pay value above $\$ 2000$, CA and TCA were most cost-effective. Although this values are high, they are still within the WHO threshold limit for cost-effectiveness $[19,20]$.

The findings of this study were similar to those reported in a Greek study of the cost-effectiveness analysis of a triple combination of valsartan, A, and hydrochlorothiazide over its dual combinations [21]. In the study, Stafylas et al. found that the single triple combination therapy was more cost-effective compared to the dual combinations (valsartan and A, A and hydrochlorothiazide, and valsartan and hydrochlorothiazide). Even though the triple combination therapy was the most expensive, it is a cost-effective choice for patients with moderate to severe hypertension [21]. 
Table 3: Probability of BP control and cost-effectiveness of a triple antihypertensive therapy and 3 different dual combinations

\begin{tabular}{|c|c|c|c|c|}
\hline \multirow[t]{2}{*}{ Variables } & \multicolumn{4}{|c|}{ Study group (mean \pm standard deviation) } \\
\hline & TCA & TC & CA & TA \\
\hline Cost of drugs per annum (\$) & $26.24 \pm 0.05$ & $23.86 \pm 0.03$ & $21.47 \pm 0.04$ & $17.89 \pm 0.03$ \\
\hline EQ-5D index score (utility valuation) & $0.78 \pm 0.10$ & $0.74 \pm 0.12$ & $0.80 \pm 0.13$ & $0.69 \pm 0.11$ \\
\hline Probability of BP control & $0.37 \pm 0.01$ & $0.23 \pm 0.02$ & $0.21 \pm 0.01$ & $0.19 \pm 0.01$ \\
\hline QALY & $0.128 \pm 0.0064$ & $0.126 \pm 0.0073$ & $0.130 \pm 0.0075$ & $0.123 \pm 0.0058$ \\
\hline Cost per QALY & $205.00 \pm 8.69$ & $189.37 \pm 9.35$ & $165.15 \pm 7.14$ & $145.45 \pm 7.1$ \\
\hline Cost per BP control & $70.92 \pm 0.04$ & $103.74 \pm 0.06$ & $102.24 \pm 0.03$ & $94.16 \pm 0.05$ \\
\hline
\end{tabular}

TCA: Telmisartan+Chlorthalidone+Amlodipine; TC: Telmisartan+Chlorthalidone; CA: Chlorthalidone+Amlodipine, TA: Telmisartan+Amlodipine, BP: Blood pressure, QALY: Quality adjusted life years, EQ-VAS: EQ visual analogue scale

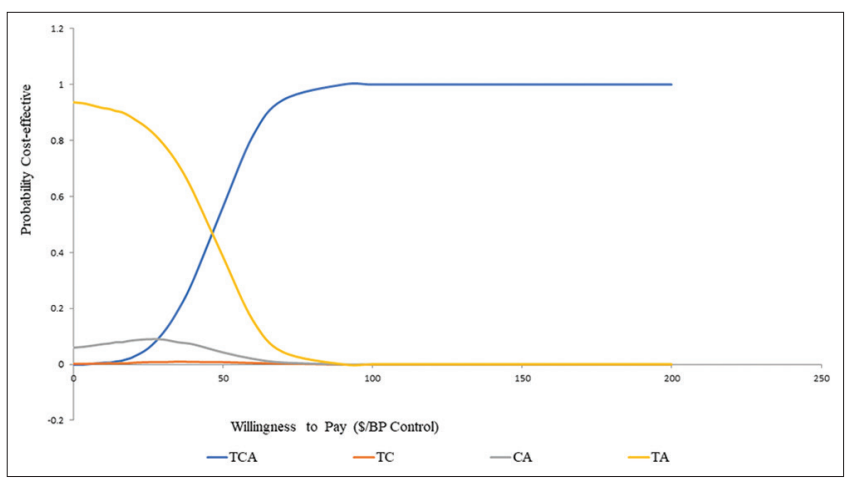

Fig. 1: Cost-effectiveness acceptability curve for the four interventions based on cost per blood pressure control

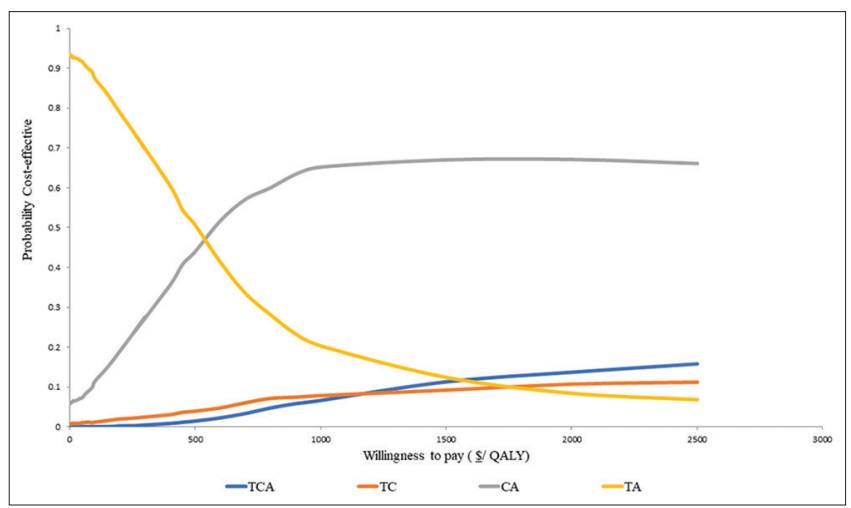

Fig 2: Cost-effectiveness acceptability curve for the four interventions based on cost per quality adjusted life years

Although diuretics generally appear to be the least expensive antihypertensive drug class, they are usually recommended as monotherapy for hypertensive patients without any compelling indication. Nevertheless, studies have shown that hypertensive patients require an average of three drugs for effective BP control [9]. The present study lends support to this notion. It has been suggested that in black patients who require more than one drug for BP control, a combination of renin-angiotensin-aldosterone system inhibitor, dihydropyridine-type calcium channel blocker, and diuretic should be recommended.

In this study, the triple combination was the most cost-effective in terms of BP control. The probability of achieving target BP control was about 2 times higher for patients on triple combination compared to those taking dual combinations. Nevertheless, $\mathrm{T}$ and A combinations should be reserved as an alternative considering that patients benefitted more from the combination in terms of cost per QALY gained. Hence, in cases where the quality of life of the patient is of paramount importance, $\mathrm{T}$, and A combination should be considered as the first option. Furthermore, patients who are unable to afford the triple combination therapy can equally benefit from the TA dual combination as it is the least expensive.

Furthermore, the current treatment guidelines advocate for the use of single fixed dose combinations for patients requiring more than one antihypertensive drug to attain optimal BP control [10]. This is because of the high prevalence of poor medication adherence often seen among patients on multiple medications. The use of fixed dose combinations improves medication adherence with resultant better BP control and reduction in cardiovascular risks associated with hypertension $[14,22]$.

In addition, inappropriate antihypertensive combination or poor drug selection is thought to be responsible for low BP control in many countries. A large proportion of patients present to the hospitals or clinics with resistant hypertension, thus giving a unique challenge to the clinician regarding the best drug combination to prescribe bearing in mind patient's characteristics and financial strength. Thus, there is need for fixed dose combinations.

The parameters of the laboratory tests (pre and post) carried out showed that the drug combinations had no significant effect on the electrolytes and other key parameters.

The findings of the present study would serve as possible guide for clinical practitioners in Nigeria; regarding the clinical and economic efficacies of commonly recommended antihypertensive drug combinations for the Black population.

\section{Limitations}

One major limitation of this study was that we used a small sample size from three settings in one town. Using a larger population and more centers are recommended in future studies.

Furthermore, extra laboratory tests would have given us a clearer effect of some pharmacodynamic properties of the different drug combinations, for example, liver function test and platelet count. There is growing evidence that thiazides and thiazide-like diuretic reduces platelet aggregation. However, the extent is still unknown and investigations are still ongoing $[23,24]$. Conducting platelet count test would have provided information about their effect on platelet count.

\section{CONCLUSION}

The triple combination therapy of TCA had the best cost per BP control followed by TA combination in the management of hypertensive patients. Furthermore, triple combination therapy demonstrated the highest probability of BP control. Therefore, this study provides valuable information to guide clinicians in decision-making regarding appropriate antihypertensive drug selection in the management of hypertensive patients. However, there is a need to further validate the study findings using larger population. 


\section{ACKNOWLEDGMENT}

The authors are grateful to the nurses (Mrs. Idoko Leticia, Mrs. Ugwu Scholastica, Mrs. Perpetua Eze and Mrs. Ugwu Theresa) for their support in collection of patients' data.

\section{AUTHORS' CONTRIBUTIONS}

Adibe MO (Methodology, Formal Analysis, Writing - Review and Editing and Validation) Ukwe CV (Conceptualization, Project Administration and Supervision), Okafor CE (Funding Acquisition), Anosike C (Data Curation, Writing - Original Draft Preparation and Visualization), Isah A (Data Curation, software and resources), Ike SO (Investigation), Anisiuba BC (Investigation), Nwuruku, GC (Investigation).

\section{CONFLICTS OF INTEREST}

There are no conflicts of interest.

\section{FUNDING}

Funding for this project was provided by Nemel Pharmaceuticals Limited. The views expressed in this article are those of the authors, and no official endorsement by Nemel Pharmaceuticals Limited is intended or should be inferred.

\section{REFERENCES}

1. World Health Organization. A Global Brief on Hypertension-silent Killer, Global Public Health Crisis: World Health Day 2013. Geneva, Switzerland: World Health Organization; 2015.

2. Ogah OS, Adebiyi AA, Oladapo OO, Adekunle AN, Oyebowale OM, Falase AO, et al. The changing patterns of heart disease in Nigeria: Data from the Ibadan outpatient cardiac registry. Circulation 2012;125:e673.

3. Ogah OS, Okpechi I, Chukwuonye II, Akinyemi JO, Onwubere BJ, Falase AO, et al. Blood pressure, prevalence of hypertension and hypertension related complications in Nigerian Africans: A review. World J Cardiol 2012;4:327-40.

4. Gradman AH, Parisé H, Lefebvre P, Falvey H, Lafeuille M, Duh MS. Initial combination therapy reduces the risk of cardiovascular events in hypertensive patients: A matched cohort study. Hypertension 2012;61:309-18

5. Kearney PM, Whelton M, Reynolds K, Muntner P, Whelton PK, He J. Global burden of hypertension: Analysis of worldwide data. Lancet 2005;365:217-23.

6. Adeloye D, Basquill C, Aderemi AV, Thompson JY, Obi FA. An estimate of the prevalence of hypertension in Nigeria: A systematic review and meta-analysis. J Hypertens 2014;32:1-13.

7. World Health Organization. WHO Bulletin: Nigeria Wake Up to High Blood Pressure. Geneva: World Health Organization; 2013.

8. Salako B, Ayodele O, Kadiri S, Arije A. Assessment of blood pressure control in a black African population. Trop Cardiol 2002;28:3-6

9. Gradman AH. Rationale for triple-combination therapy for management of high blood pressure. J Clin Hypertens (Greenwich) 2010;12:869-78.

10. Canbakan B. Rational approaches to the treatment of hypertension: Drug therapy-monotherapy, combination, or fixed-dose combination? Kidney Int Suppl (2011) 2013;3:349-51.

11. Wald DS, Law M, Morris JK, Bestwick JP, Wald NJ. Combination therapy versus monotherapy in reducing blood pressure: Meta-analysis on 11,000 participants from 42 trials. Am J Med 2009;122:290-300.

12. Dennison-Himmelfarb C, Handler J, Lackland DT. 2014 Evidencebased guideline for the management of high blood pressure in adults report from the panel members appointed to the eighth joint national committee (JNC 8). JAMA 2014;1097:1-14.

13. Kalra S, Kalra B, Agrawal N. Combination therapy in hypertension : An update. Diabetol Metab Syndr 2010;2:44.

14. Chobanian AV, Bakris GL, Black HR, Cushman WC, Green LA, Joseph LJ, et al. The seventh report of the joint national committee on prevention, detection, evaluation, and treatment of high blood pressurethe JNC 7 report. JAMA 2003;289:2560-71.

15. Wright JM, Musini VM, Gill R. First-line drugs for hypertension. Cochrane Database Syst Rev 2018;4:CD001841.

16. Harman J, Walker ER, Charbonneau V, Akylbekova EL, Wyatt SB. Treatment of hypertension among African Americans: The Jackson heart study. J Clin Hypertens (Greenwich) 2014;15:367-74.

17. Ekwunife OI, Ezenduka CC, Uzoma BE. Evaluating the sensitivity of EQ-5D in a sample of patients with Type 2 diabetes mellitus in two tertiary health care facilities in Nigeria. BMC Res Notes 2016;9:24.

18. Murray C, Lauer J, Niessen L, Tomijima N, Rodgers A. Effectiveness and costs of intervention to lower systolic blood pressure and cholesterol: A global and regional analysis on reduction of cardiovascular-disease risk. Lancet 2003;361:717-25.

19. Edejer TT, Baltussen R, Adam T, Hutubessy R, Acharya A, Evans DB, et al. WHO Guid to Cost-effectiveness Analysis. Geneva: World Health Organization; 2003.

20. Woods B, Revill P, Sculpher M, Claxton K. Country-level costeffectiveness thresholds: Initial estimates and the need for further research. Value Health 2016;19:929-35.

21. Stafylas P, Kourlaba G, Hatzikou M, Georgiopoulos D, Sarafidis P, Maniadakis N. Economic evaluation of a single-pill triple antihypertensive therapy with valsartan, amlodipine, and hydrochlorothiazide against its dual components. Cost Eff Resour Alloc 2015;13:10.

22. James PA, Oparil S, Carter BL, Cushman WC, Dennison-Himmelfarb C, Handler J, et al. 2014 Evidence-based guideline for the management of high blood pressure in adults: Report from the panel members appointed to the eighth joint national committee (JNC 8). JAMA 2014;311:507-20.

23. Rosendorff C. Why are we still using hydrochlorothiazide? J Clin Hypertens (Greenwich) 2011;13:10-2.

24. Khalid B, Michael W, Daniel EH, Venkata MA, Venkata A, Tammy LB. Chlorthalidone and HCTZ Impacts on Platelet Activation. United States: Clinical Trial; 2014 